\title{
DIETMAR TREIBER
}

\section{A non-archimedean "curve integral" and its application to the construction of potentials and solutions of differential equations}

Mémoires de la S. M. F., tome 39-40 (1974), p. 359-374

<http://www.numdam.org/item?id=MSMF_1974_39-40_359_0>

(C) Mémoires de la S. M. F., 1974, tous droits réservés.

L'accès aux archives de la revue "Mémoires de la S. M. F. » (http://smf. emath.fr/Publications/Memoires/Presentation.html) implique l'accord avec les conditions générales d'utilisation (http://www.numdam.org/conditions). Toute utilisation commerciale ou impression systématique est constitutive d'une infraction pénale. Toute copie ou impression de ce fichier doit contenir la présente mention de copyright.

\section{Numdam}


A NON-ARCHIMEDEAN "CURVE INTEGRAL" AND ITS

APPLICATION TO THF CONSTRUCTION OF POTENTIALS

AND SOLUTIONS OF DIFFFRFNTTAL EQUATIONS

Dietmar TREIBER

In the opinion of many students integration is the conversion of differentiation. That means more exactly : Integration serves for the construction of primitive functions and potentials. In this article it will be shown that there is a procedure of constructing potentials in n.a. Banach spaces, which is similar to that of the archimedean case by means of the curve integral.

At first let us remember the corresponding statements and the main ideas of the proofs in the archimedean case:

Let $G$ be an open subset of $\mathbb{R}^{n}$ and let

$$
\omega: G \longrightarrow \mathscr{L}\left(\mathbb{R}^{n}, \mathbb{R}\right)
$$

be a differential form on $G$, that means : $\omega$ is a mapping from $G$ into the Banach space of all (bounded) linear maps from $\mathbb{R}^{n}$ to $R$. We regard the problem whether there exists a "potential of $\omega$ ", that is a differentiable function from $G$ to $\mathbb{R}$, whose derivative equals to the given $\omega$. The following two conditions are sufficient for the existence of a potential of $\omega$ :

1) G is simply connected ;

2) $\omega$ is differentiable and closed.

If $\omega$ is differentiable, then the closedness of $\omega$ is also a necessary condition for the existence of a potential of $\omega$, as is known by the theorem about the equality of the mixed second partial derivatives.

A construction of a potential of $\omega$ can be given by means of the curve integral, especially in the case. $G=\mathbb{R}^{n}$ in the following way :

For $v \in R^{n}$ define the curve $c(v)$ by

$$
c(v)(t):=t v, 0 \leqslant t \leqslant 1 \text {. }
$$

If condition (2) for $\omega$ is fulfilled, then the function 


$$
v \longmapsto \int_{c(v)} \omega
$$

defines a potential of $\omega$.

In the n. a. case the special problem of constructing primitive functions to continuous functions over the fields $Q_{p}$ has already been solved by Dieudonné [1] in his classical work of 1944. Here the construction of primitive functions appears as a special case of a theorem about the existence of solutions of certain p-adic differential equations. In 1967 van der PUT [4] gave a method of solving p-adic differential equations by means of an orthogonal base of the Banach space of all continuous functions from $\mathbb{Z}_{p}$ to a $n$. a. completely valued field. $K \supseteq \mathbb{Q}_{p}$.

It is remarkable that all mentioned researches about the existence of solutions of $n$. a. differential equations are restricted to the fields $Q_{p}$. The reason : The elements of $Q_{p}$ allow a very useful development into infinite series. Our first aim is therefore to show that there exists a similar development into infinite series for the elements of all $n$. a. Banach spaces.

The procedure of constructing primitive functions over $\mathbb{Q}_{\mathrm{p}}$, which one gets as a special case in the works of Dieudonné, van der PUT and in this work, is essentially the same.

We use in this work the following notations :

i) $\mathbb{R}, \mathbb{R}, \mathbf{N}:=$ real, integer, natural numbers (including 0 ); $\mathbf{R}_{+}$resp. $\mathbb{N}_{+}:=$positive real resp. natural numbers.

i.i) Let $K$ be a $n$. a. non-trivial completely valued field.

iii) Let $E$ and $E^{\prime}$ be $n$. a. Banach spaces over $K$.

\section{Development of the elements of a $\mathrm{n}$. a. Banach space into infinite series}

1.1. Definition. Let $\left(u_{i}\right)_{i} \in \mathrm{z}$ be a sequence of elements of a vector space $V$ over K. Then we call $\left(u_{i}\right)_{i \in \mathbf{z}}$ left-finite iff there exists an $n \in Z$ such that $u_{i}=0 \in V$ for all i $\in$ with $i<n$.

1.2. Lemma. Let $E$ be a n. a. Banach space over $K$. Assume a $\in K$ such that $0<|a|<1$. Then there exists a subset $\mathrm{R}$ of $\mathrm{E}$ with the following properties :

i) For every left-finite sequence $\left(u_{i}^{\prime}\right)_{i \in Z}$ of elements of $R$ the series

$$
\sum_{i=-\infty}^{+\infty} u_{i} a^{i}
$$

converges in $\mathrm{E}$. 
ii) Every element u $\epsilon E$ has a representation of the form

$$
u=\sum_{i=-\infty}^{+\infty} u_{i} a^{i}
$$

with a left-finite sequence $\left(u_{i}\right)_{i \in z}$ of elements of $R$.

iii) Let $u=\sum u_{i} a^{i}, v=\sum v_{i} a^{i}$ be elements of $R$, represented as limits of infinite series in the sense of (ii). Assume

$$
\mathrm{n}:=\inf \left\{i \in \mathrm{z} \mid \mathrm{u}_{i} \neq \mathrm{v}_{i}\right\} \in \mathrm{z} \text {. }
$$

Then :

$$
|a|^{n+1}<H u-v\|=\| u_{n}-v_{n} \| \cdot|a|^{n} \leqslant|a|^{n} .
$$

This implies especially that the representation of the elements of $\mathrm{E}$ in the sense of (ii) is unique.

Proof : Let $R$ be a system of representatives of

$$
\{u \in E \mid\|u\| \leqslant 1\} /\{u \in E|\|u\| \leqslant| a \mid\}
$$

with 0 € R. Clearly (i) holds.

Assume $u \in E, u \neq 0$. Then there exists an $n \in \mathbb{Z}$ such that

$$
|a|^{n+1}<\|u\| \leqslant|a|^{n} .
$$

It follows :

$$
|a|<\left\|\frac{1}{a^{n}} u\right\| \leqslant 1 .
$$

Then there exists an $u_{n} \in R$ such that :

$$
\left\|u_{n}-\frac{1}{a^{n}} u\right\| \leqslant|a| .
$$

Therefore :

$$
\left\|u-u_{n} a^{n}\right\| \leqslant|a|^{n+1} .
$$

By iterating this process we get (ii). Part (iii) is a consequence of the strong triangle inéquality 
2. The curve integral and its application to the construction of potentials

2. 1. Definition. Let $\mathscr{L}\left(E, E^{\prime}\right)$ be the vector space of all continuous linear mappings from $E^{\text {to }} E^{\prime}$. Then $\mathcal{L}\left(E, E^{\prime}\right)$ is a $n$. a. Ban space relative to the norm

$$
\|1\| \quad:=\sup \left\{\frac{\|1(\mathrm{v})\|}{\|v\|} \mid v \in E, \quad v \neq 0\right\}, \quad 1 \in \mathcal{L}\left(\mathrm{E}, \mathrm{E}^{\prime}\right) \text {. }
$$

Assume that $G$ is an open subset of $E$. A map

$$
\omega: G \longrightarrow \mathscr{L}\left(E, E^{\prime}\right)
$$

is called a differential form (or 1-form) on $G$ (with coefficients in $E^{\prime}$ ).

Let $\omega: G \longrightarrow \mathcal{L}\left(E, E^{\prime}\right)$ be a differential form on $G$. A function $f: G \longrightarrow E^{\prime}$ is called a potential of $\omega$ iff $f$ is differentiable on $G$ and for all $p \in G$ the derivative of $f$ at $p$ equals to $\omega(p)$.

2.2. Definition. Let $G$ be an open subset of $E$ and

$$
\omega: G \longrightarrow \mathcal{L}\left(E, E^{\prime}\right)
$$

be a continuous differential form on $G$.

i) A curve $c$ in $E$ is a mapping

$$
\mathrm{c}: \mathrm{Z} \longrightarrow \mathrm{E} \text {, }
$$

such that there exist the limits $\lim _{n \rightarrow-\infty} c_{n} \underset{n \rightarrow+\infty}{\text { and }} \lim c_{n}$. We call the left limit of $c$ the "initial point of $c "$, the right limit of $c$ the "end point of $c "$.

ii) A curve $c: \mathbb{Z} \longrightarrow E$ is called curve in $G$ iff the image set of $c$, the initial point of $c$ and the end point of $c$ lie in $G$.

iii) Let $c: \mathbb{Z} \longrightarrow E$ be a curve in $G$. We define the integral of $\omega$ over the curve $\dot{c}$ as :

$$
\int_{c} \omega:=\sum_{n=-\infty}^{+\infty} \omega\left(c_{n}\right)\left(c_{n+1}-c_{n}\right) .
$$

Remember that $\omega\left(c_{n}\right)\left(c_{n+1}-c_{n}\right)$ is the image of $\left(c_{n+1}-c_{n}\right) \in E$ under the $\operatorname{map} \omega\left(c_{n}\right) \in \mathscr{L}\left(E, E^{\prime}\right)$.

2.3. Proposition: Let $G$ be an open subset of $E$ and $c: \mathbb{Z} \longrightarrow$ be a curve in $G$. Then :

i) The curve integral defined in 2.2. is K-1inear, that means : 


$$
\int_{c} \cdot(a \omega+b \tilde{\omega})=a \int_{c} \omega+b \int_{c} \tilde{\omega}
$$

ii) The curve integral is continuous, that means : Let $\left(\omega_{I}\right)_{I \in N}$ be a sequence of continuous mappings from $G$ to $\mathcal{Z}\left(E, E^{\prime}\right)$, which converges locally uniform to a continuous differential form $\omega$ on $G$. Then the sequence $\left(\int_{c} \omega_{1}\right) \in \mathbb{N}$ converges to $\int_{c} \omega$.

iii) Let $\left(\omega_{1}\right)_{1 \in N}$ be a sequence of continuous differential forms on $G$ with. coefficients in $\mathrm{E}^{\prime}$, which is uniformly bounded and converges (pointwise) to a continuous differential form $\omega$ on $G$. Then $\left(\int_{c} \omega_{I}\right)_{1 \in \mathbb{N}}$ converges to $\int_{c} \omega$.

Proof : simple application of the definitions.

2.4. Definition. Assume $u=\sum_{i=-\infty}^{+\infty} u_{i} a^{i} \in E$. Define a curve $c(u): z \rightarrow E$ by :

$$
c(u)_{n}:=\sum_{i=-\infty}^{n} u_{i} a^{i}
$$

for all $n \in \mathbb{z}$. Obviously the initial point of $c(u)$ is 0 , its end point is $u$. Furthermore :

$$
u=\sum_{n=-\infty}^{+\infty}\left(c(u)_{n+1}-c(u)_{n}\right) .
$$

2.5. Lemma. If

$$
\omega: \mathrm{E} \longrightarrow \mathcal{L}\left(\mathrm{E}, \mathrm{E}^{\prime}\right)
$$

is a continuous differential form, it holds for all $u, v \in E$ :

$\left\|\int_{c(u)} \omega-\int_{c(v)} \omega\right\|^{\prime} \leqslant \sup \left\{\|\omega(p)\| \mid\|p-v\| \leqslant \frac{1}{|a|}\|u-v\|\right\} \cdot\|u-v\|$

Proof : Assume $u, v \in E, u \neq v, u=\sum_{i=-\infty}^{+\infty} u_{i} a^{i}, v=\sum_{i=-\infty}^{+\infty} v_{i} a^{i}$.

Then there exists a minimal $\mathbb{N} \in \mathbb{Z}$ such that

$$
\mathrm{u}_{\mathrm{N}} \neq \mathrm{v}_{\mathrm{N}}
$$


Therefore :

$$
\begin{aligned}
\left\|\int_{c(u)} \omega-\int_{c(v)} \omega\right\|^{\prime} & =\| \omega\left(c(u)_{N-1}\right)\left(c(u)_{N}-c(v)_{N}\right) \\
& +\sum_{n=N}^{\infty} \omega\left(c(u)_{n}\right)\left(c(u)_{n+1}-c(u)_{n}\right) \\
& -\sum_{n=N} \omega\left(c(v)_{n}\right)\left(c(v)_{n+1}-c(v)_{n}\right) \|^{\prime}
\end{aligned}
$$

Now the statement follows with the aid of the following inequalities ; $(n \in \mathbb{Z}, n \geqslant N)$

$$
\begin{aligned}
& \left\|c(u)_{N-1}-v\right\| \leqslant \frac{1}{|a|}\|u-v\| ; \\
& \left\|c(u)_{n}-v\right\|,\left\|c(v)_{n}-v\right\| \leqslant\|u-v\| ; \\
& \left\|c(u)_{N}-c(v)_{N}\right\| \leqslant\|u-v\| ; \\
& \left\|c(u)_{n+1}-c(u)_{n}\right\|,\left\|c(v)_{n+1}-c(v)_{n}\right\| \leqslant\|u-v\| .
\end{aligned}
$$

2.6. Theorem. Let

$$
\omega: E \longrightarrow \mathcal{L}\left(E, E^{\prime}\right)
$$

be a continuous differential form on $E$ with coefficients in $E^{\prime}$. Then the map $F=E \longrightarrow E^{\prime}$, defined by

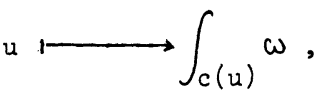

\section{is a potential of $\omega$.}

Proof : The statement is a consequence of the following inequality :

$$
\begin{aligned}
\| F(u)-F(v)- & \omega(v)(u-v) \| \cdot \\
& \leqslant \sup \left\{\|\omega(p)-\omega(v)\| \mid\|p-v\| \leqslant \frac{1}{|a|}\|u-v\|\right\} \cdot\|u-v\|
\end{aligned}
$$

for all $u, v \in E$. Proof of this inequality for $u, v \in E$; 


$$
\begin{aligned}
F(u):-F(v)-\omega(v)(u-v) & \\
=F(u)-F(v) & -\omega(v)\left[\sum_{n=-\infty}^{+\infty}\left(c(u)_{n+1}-c(u)_{n}\right)\right] \\
+ & \omega(v)\left[\sum_{n=-\infty}^{+\infty}\left(c(v)_{n+1}-c(v)_{n}\right)\right] .
\end{aligned}
$$

Because of the continuity of $\omega(v): E \longrightarrow E^{\prime}$ we conclude :

$$
\begin{aligned}
&=F(u)-F(v)-\sum_{n=-\infty}^{+\infty} \omega(v)\left(c(u)_{n+1}-c(u)_{n}\right) \\
&+\sum_{n=-\infty}^{+\infty} \omega(v)\left(c(v)_{n+1}-c(v)_{n}\right) \\
&=\int_{c(u)}[\omega-\omega(v)]-\int_{c(v)}[\omega-\omega(v)] .
\end{aligned}
$$

Now the statement follows from 2.5.

2.7. Definition. Let $G$ be an open subset of $\mathrm{E}$. We now define a mapping $\Phi_{G}$, which orders to every continuous differential form $\omega$ on $G$ with coefficients in $E^{\prime}$ a potential $\phi_{G}(\omega)$ of $\omega$ :

Let $\omega$ be a continuous differential form on $G$ :

(1) If $G=E$, let $\phi_{G}(\omega)$ be the map

$$
u \longmapsto \int_{c}(u) \omega,
$$

which has been regarded in 2.6 .

(2) If $G$ is an "open" bail in $E$, order to $\omega$ a continuous differential form $\tilde{\omega}$ on $E$ by setting $\tilde{\omega}$ equal to zero outside $G$ and equal to $\omega$ on G. Define :

$$
\Phi_{G}(\omega):=\Phi_{E}(\tilde{\omega}) \mid G .
$$

(3) If $G$ is an arbitrary open subset of $E$, which is different from $E$, then there is a canonical family $\left(U_{i}\right)_{i \in I}$ of "open" balls in $G$, disjoint two by two, such that $G$ is the union of the $U_{i}$. Define : 


$$
\Phi_{G}(\omega) \mid U_{i}^{:}:=\Phi_{U_{i}}\left(\omega \mid U_{i}\right)
$$

for all i $\in I$.

2.8. Theorem. Assume that $G$ is an open subset of $E$. Then the map $\phi_{G}$ (defined in 2.7) has the following properties :

(i) $\phi_{G}$ orders to every continuous differential form on $G$ (with coefficients in $\left.E^{\prime}\right)$ a potential ;

(ii) $\phi_{G}$ is $K$-linear :

(iii) $\phi_{G}$ is continuous relative to the canonical topologies of its definition set and image set (induced by the locally uniform convergence).

(iv) Let $\left(\omega_{l}\right) l \in$ 'N be a sequence of differential forms on $G$, which is uniformly bounded and converges (pointwise) to a continuous differential form $\omega$ on $G$, then the sequence $\left(\phi_{G}\left(\omega_{l}\right)\right)_{l \in N}$ converges (pointwise) to $\phi_{G}(\omega)$.

(v) For every continuous differential form $\omega$ on $G$ the potential $G(\omega)$ is locally extension-bounded. Example 3.2. will show that there are infinite times differentiable functions, which have not this property.

(vi) The definition of the curve integral and - as a consequence - our construction of potentials depend on the choice of a development into infinite series of the elements of $\mathrm{E}$ in the sense of 1.2. The mapping $\phi_{G}$ is not characterized by its properties.

Proof : Applications of the preceding statements.

'9. Corollary 1. The theorem about the equality of the mixed second partial derivatives does not hold in the n. a. case, more exactly :

There exists an infinite times differentiable function $f: K^{2} \rightarrow K$, such that for all $\mathrm{p} \in \mathrm{K}^{2}$ :

$$
\frac{\partial^{2} f}{\partial y \partial x}(p) \neq \frac{\partial^{2} f}{\partial x \partial y}(p) .
$$


Proof : Define $f$ by :

$$
f:=\phi_{K^{2}}(x d x+x d y): K^{2} \longrightarrow K .
$$

Then we get :

$$
\frac{\partial f}{\partial x}=\frac{\partial f}{\partial y}=x \text {, }
$$

therefore :

$$
\frac{\partial^{2} f}{\partial y \partial x}=\operatorname{const}(0) ; \frac{\partial^{2} f}{\partial x \partial y}=\operatorname{const}(1) .
$$

2. 10. Corollary 2. Let $G$ be an open subset of $E$ and $f: G \longrightarrow E^{\prime}$ be a continously differentiable function on $G$. Then $f$ can be represented as the sum of two functions $g, h: G \longrightarrow E^{\prime}$ such that :

(1) g is differentiable and locally extension-bounded on G ;

(2) $\mathrm{h}$ is differentiable on $\mathrm{G}$ with everywhere vanishing derivative.

2.11. Remark. Assume $\operatorname{Char}(K)=0, G$ an open subset of $K$. The question arises whether exists a function $\phi$, which orders to every continuous function from $G$ to $K$ a primitive function and furthermore has the following properties :

(1) $\phi$ orders to the function $x^{n} \mid G$ the primitive function $\frac{1}{n+1} x^{n+1} / G(n \in N)$;

(2) $\Phi$ is K-Iinear ;

(3) $\Phi$ is continuous (relative to the canonical topology of the space of all continuous functions from $\mathrm{G}$ to $\mathrm{K}$, which is induced by the locally uniform convergence).

The function $\Phi_{G}$ defined in 2.7. is linear and continuous. But it has not the property (1) : For instance the primitive function of $\mathrm{x}: \mathrm{K} \longrightarrow \mathrm{K}$, which one calculates using the method described above is the following :

$$
\frac{1}{2} x^{2}-g
$$


where $\mathrm{g}: \mathrm{K} \longrightarrow \mathrm{K}$ is defined by

$$
g\left(\sum_{n=-\infty}^{+\infty} a_{n} a^{n}\right):=\frac{1}{2} \sum_{n=-\infty}^{+\infty}\left(a_{n} a^{n}\right)^{2} .
$$

The following theorem will show that the properties (1) and (3) are not independent and that from this reason a function as described above generally does not exist.

2.12. Theorem. Assume $\operatorname{Char}(K)=0$ and that the restriction of the valuation of $K$ to $Q$ is non-trivial. Let $G$ be an open subset of $K$ and let $C^{\circ}(G, K)$ denote the space of all continuous functions from $G$ to $K$. Then generally it does not exist a mapping

$$
\phi: C^{\circ}(G, K) \longrightarrow C^{\circ}(G, K),
$$

which is continuous and fulfills

$$
\phi\left(x^{n} \mid G\right)=\frac{1}{n+1} \cdot x^{n+1} \mid G
$$

for all $n \in \mathbb{N}$.

Proof : (cf. Dieudonné [1]) Let $G$ be the "open" ball in $E$ of radius 1 around the center 0 . Assume that $\phi$ is a mapping as described above. Let $p$ be the unique prime number such that the restriction of the valuation of $K$ to $Q$ is equivalent to the p-adic valuation of $\mathbb{Q}$.

Put :

$$
f_{n}:=p^{n} x^{p^{2 n}}-1 / G
$$

for all $n \in \mathbb{N}$. Then $\left(f_{n}\right)_{n \in \mathbb{N}}$ converges to the zero-function on $G$, but $\left(\phi\left(f_{n}\right)\right)_{n \in N}$ does not converge at all (Convergence relative to the canonical topology of $\left.\mathrm{C}^{\circ}(\mathrm{G}, \mathrm{K})\right)$.

Remark. Assume $\mathrm{k} \in \mathrm{N}, \mathrm{k}>1, \mathrm{G}$ an open subset of $\mathrm{E}$. In the same way as in the real analysis one can define k-differential forms on $G$ with coefficients in $E^{\prime}$ also in the $n$. a. case. Using the preceding results it is easy to show that in this case too every continuous $k$-differential form on $G$ has a potential. 


\section{Examples.}

The examples of this section shall illustrate the connection between the notions of continuity, (partial) differentiability and existence of primitive functions. Furthermore they show some consequences of the lack of a mean value theorem in the n. a. analysis.

3.1. Example. (cf. Dieudonné [1]) There exists an injective function $f: E \longrightarrow E$, which is differentiable on $E$ and whose derivative is vanishing everywhere.

Proof : Define $f$ by

$$
f\left(\sum_{n=-\infty}^{+\infty} v_{n} a^{n}\right):=\sum_{n=-\infty}^{+\infty} v_{n} a^{2 n} .
$$

We get the following inequality : For all $\mathrm{v}, \mathrm{w} \in \mathrm{E}$ :

$$
\left\|_{v}-w\right\|^{2} \leqslant\|f(v)-f(w)\|^{\prime} \leqslant \frac{1}{|c|}\|v-w\|^{2}
$$

3.2. Example. There exists a function $f: E \longrightarrow K$, which is differentiable on $E$ with everywhere vanishing derivative, but which is not locally extension-bounded.

Proaf : The valuation of $K$ is non-trivial. Therefore there exists an a $\epsilon \mathrm{K}$ such that $0<\mid$ a $\mid<1$. Assume $v \in \mathbb{E},\|v\| \geqslant 1$. Then we can find a map $i: \mathbb{N} \longrightarrow \mathbb{N}$ such that

$$
|a|^{i(n)}<\min \left\{|a|^{n}, \frac{i}{(n+1) \cdot\|v\|}|a|^{2 n}\right\}
$$

for all $n \in \mathbb{N}$. Define for $n \in \mathbb{N}$ :

$$
p_{n}:=a^{n} v, q_{n}:=a^{n} v+a^{i(n)} v
$$

Let $U_{n}$ be the "open" ball of radius $|a|^{i(n)}\|v\|$ around $a^{n} v$ and $X_{n}$ its characteristic function. Define :

$$
f:=\sum_{n=0}^{\infty} e^{2 n} x_{n} .
$$


Obviously $\mathrm{f}$ is differentiable on E. with everywhere vanishing derivative.

Furthermore :

$$
\left|f\left(p_{n}\right)-f\left(q_{n}\right)\right| \geqslant(n+1) \cdot\|v\| \cdot a^{i(n)}=(n+1)\left\|p_{n}-q_{n}\right\| .
$$

3.3. Example. There exists a function $f: K^{2} \longrightarrow K$, which is partially differentiable on $\mathrm{K}^{2}$ and whose partial derivatives are vanishing everywhere, but which is not continuous in 0 . Especially continuous partial differentiability does not imply continuity.

Proof: Assume a $\in \mathrm{K}, 0<\mid$ a $\mid<1$. For all $\mathrm{n} \in \mathbb{N}$ let $U_{\mathrm{n}}$ be the "open" ball in $\mathrm{K}^{2}$ of radius $|a|^{n}$ around the center $\left(a^{n}, a^{n}\right)$. Define $f$ as the characteristic function of the union of the $U_{n}$.

3.4. Example. There exists a function $f: K \longrightarrow K$, which is extension-bounded on $K$ and therefore equicontinuous on $\mathrm{K}$, but which is nowhere differentiable on $\mathrm{K}$.

Proof : Define $f$ by :

$$
f\left(\sum_{n=-\infty}^{+\infty} a_{n} a^{n}\right):=\sum_{n=-\infty}^{+\infty} a_{2 n} a^{2 n} .
$$

Obviously it holds for all b,d $\in \mathrm{K}$ :

$$
|f(b)-f(d)| \leqslant|b-d|
$$

thus $f$ is extension-bounded on K. Assume

$$
b=\sum_{n=-\infty}^{+\infty} b_{n} a^{n} \in K .
$$

Let $R$ be the subset of $K$ defined in 1.2. Then $R$ has at least two elements. Therefore there exists for all $j \in \mathbb{N}$ an

$$
\tilde{b}_{j} \in R-\left\{b_{j}\right\}
$$

Define $d_{j}:=b-b_{j} a^{j}+\tilde{b}_{j} a^{j} \in K$. Then we get $a_{j} \neq b$ and 


$$
\begin{aligned}
& \qquad \frac{f\left(d_{j}\right)-f(b)}{d_{j}-b}=\left\{\begin{array}{l}
1, \text { if } j \text { is even, } \\
0, \text { if } j \text { is odd. }
\end{array} \text { Furthermore : } \lim _{j \rightarrow \infty} d_{j}=b, \text { therefore : } f \text { is not differentiable at } b\right. \text {. }
\end{aligned}
$$

3.5. Example. There exists a function $f: K \longrightarrow K$, which has no primitive function. Proof : Let $S$ be the set of all elements of $K$, whose development into a series in the sense of 1.2. is finite. It is easy to show that the characteristic function of $\mathrm{S}$ has no primitive function.

\section{Application of the curve integral to the solution of differential equations over n. a. Banach spaces.}

In this section we give an existence theorem about the solutions of $n$. $a$. differential equations. The proof - a construction by means of the curve integralis omitted.

4. 1. Definition. Let $G$ resp. G' be an open subset of E resp. E'. Let

$$
\Omega: G \times G^{\prime} \longrightarrow \mathscr{L}\left(\mathrm{E}, \mathrm{E}^{\prime}\right)
$$

be a mapping. We are searching solutions $f: G \rightarrow G '$ of the differential equation

$$
d_{p} f=\Omega(p, f(p))
$$

for all $p \in G$.

In the case $E=K^{m}, E^{\prime}=K^{n}\left(m, n \in \mathbb{N}_{+}\right)$we regard the differential equation

$$
\left(\begin{array}{ccc}
\frac{\partial f_{1}}{\partial x_{1}} & \cdots \cdots & \frac{\partial f_{1}}{\partial x_{m}} \\
\vdots & \vdots & \vdots \\
\vdots & & \vdots \\
\frac{\partial f_{n}}{\partial x_{1}} & \cdots \cdots & \frac{\partial n}{\partial x_{m}}
\end{array}\right)(p)=\left(\begin{array}{c}
F_{1}(p, f(p)) \\
\vdots \\
\vdots \\
F_{n}(p, f(p))
\end{array}\right)
$$


for all $p \in G$. Especially in the case $E=E^{\prime}=K$ we search for solutions of the differential equation

$$
f^{\prime}(x)=F(x, f(x)) \text {. }
$$

4.2. Theorem. Let G resp. G' be an open subset of E resp. E'. Let

$\Omega: G \times G^{\prime} \longrightarrow \mathcal{L}\left(E, E^{\prime}\right)$ be a continuous mapping. Assume that

$$
\Omega: G \longrightarrow \mathbb{R}_{+}, g: G \longrightarrow G^{\prime}
$$

are continuous functions. Let $N$ be an isolated subset of $E$. Then there exists a solution

$$
f: G \longrightarrow G^{*}
$$

of the differential equation (*) such that

$$
\|f(p)-g(p)\| \|^{\prime}<\varepsilon(p)
$$

for all $p \in G$ and moreover $f$ and $g$ have the same values on $N$.

4.3. iorollary. The set of solutions of the differential équation (*) is dense in the canonically topologized space of continuous functions from $G$ to $G^{\prime}$.

Espacially the set of potentials of a continuous differential form is dense in the space of continuous functions.

\section{Analytic solutions of n. a. differential equations.}

In the last section we have seen that the set of solutions of a n. a. differential equation is dense in the space of continuous functions. If one regards analytic n. a. differential equations and searches only for analytic solutions, one gets a uniqueness theorem. Moreover an estimation of the radius of convergence of a solution will be given which generally cannot be improved.

5.1. Theorem. Assume $\operatorname{Char}(\mathrm{K})=0$. Let

$$
F=\sum_{n, m=0}^{\infty} a_{n m} x^{n \cdot y^{m}}: K^{2} \longrightarrow K
$$

be an analytic function in a neighbourhood of 0 . Then there exists a unique analytic 
solution $f$ of the differential equation

$$
\left.f^{\prime}=F(x, f)\right)
$$

such that $f(0)=0$.

Furthermore we have the following estimation for the radius of convergence of $f$ : If $F$ converges on the "closed" ball in $K^{2}$ of radius $r \in \mathbb{R}_{+}$around 0 , then we get :

$$
\rho(f) \geqslant \rho(\exp ) \frac{r}{\max \left\{\sup _{n, m \in \mathbb{N}}\left|a_{n m}\right| r^{n+m}, 1\right\}}
$$

The exponential function and its differential equation show that this estimation generally cannot be improved.

Proof : It is easy to show that there is a unique sequence $\left(b_{n}\right)_{n} \in \mathbb{N}$ of elements of $\mathrm{K}$ such that the formal power series

$$
\sum_{n=1}^{\infty} b_{n} x^{n}
$$

fulfills the differential equation (1). Now the statement follows if the inequality (2) is proved.

Obviously for all $k \in \mathbb{N}$ the $(k+1)-t h$ formal derivative of $f$ is a sum, whose single terms are of the form

$$
\frac{\partial^{n+m}}{\partial x^{n} \partial y^{m}} F(x, f(x)) f^{\left(j_{1}\right)} \ldots f^{\left(j_{m}\right)}
$$

$\left(n, m \in \mathbb{N}_{+}, n+m \in\{1, \ldots, k\}, j_{1}, \ldots, j_{m} \in N, j_{1}+\ldots+j_{m}+n=k\right)$

Assume $r \in \mathbb{R}_{+}$according to the assumption above. Put

$$
\alpha:=\sup _{n, m \in \mathbb{N}}\left|a_{n m}\right| r^{n+m} \in \mathbb{R} .
$$

Then it holds $\mathrm{n}, \mathrm{m} \in \mathbf{N}$ :

$$
\left|\frac{\partial^{n+m}}{\partial x^{n} \partial y^{m}} F(x, f(x))(0,0)\right|=\left|n ! m ! a_{n m}\right| \leqslant \frac{\alpha}{r^{n+m}}
$$


Using (3) and (4) one proves the following inequality :

$$
\left|f^{(k+1)}(0)\right| \leqslant \frac{\max \left\{\alpha^{k}, 1\right\}}{r^{k}}
$$

for all $k \in \mathbb{N}$. Therefore and because of the wellknown formula

$$
\lim _{n \rightarrow \infty} \sqrt[n]{|n| \mid}=\rho(\exp )
$$

we get :

$$
\begin{aligned}
\lim _{n \rightarrow \infty} \sup \sqrt[n]{\left|b_{n}\right|} & =\lim _{n \rightarrow \infty} \sup \sqrt[n]{\frac{\mid f(n)}{|n !|}} \\
& \leqslant \frac{1}{\rho(\exp )} \frac{\max \{\alpha, 1\}}{r}
\end{aligned}
$$

BIBLIOGRAPHY

[1] DIEUDONNE, J. Sur les fonctions continues p-adiques. Bull. Sc. Math. 68, 79-95 (1944).

[2] LOONSTRA, F. Die Lösung von Differentialgleichungen in einem bewerteten Körper. Proc. Ned. Akad. v. Wetensch. 44, 409-419 (1941).

[3] MONNA, A.F. Analyse non-archimédienne. Berlin, Heidelberg, New York Springer 1970.

[4] van der PUT, M. Algèbres de fonctions continues p-adiques. Thesis University of Utrecht 1967.

[5] TREIBER, D. Beiträge zur nicht-archimedischen Funktional-analysis. Thesis University of Cologne 1971.

Dr. Dietmar TREIBER

Mathematisches Institut der Universität

BRD - 5000 Köln 14 Weyertal 86-90 